\title{
Recognizing, Detecting and Understanding the Development of Lipid Oxidation Off-Flavors in Foods and Beverages
}

\author{
Ray Marsili* and Charles Laskonis \\ Rockford University, USA

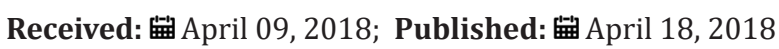 \\ *Corresponding author: Ray Marsili, Rockford University, USA
}

\begin{abstract}
Lipid oxidation is a major cause of off-flavor problems in the food industry. Lipid oxidation products can be difficult to detect and identify. New sample preparation techniques to extract lipid oxidation products prior to GC-MS analysis and innovative more sensitive GC-TOFMS instrumentation that performs peak de convolution with co eluting chromatographic peaks are critical to detecting these chemicals and diagnosing possible mechanisms of lipid oxidation. The goal of this article is to show how these new analytical technologies have been applied to solving lipid oxidation problems in milk, ice cream, onion dip, sour cream, powdered nondairy creamer, bottled water, and omega-3 fortified foods.
\end{abstract}

Keywords: Lipid oxidation products; Aldehydes; Ketones, Dimethyl disulfide; Odor synergy effects

Abbreviations: GC-TOFMS: Gas Chromatography Time-of-Flight Mass Spectrometry; DTD: Direct Thermal Desorption; PDMS: Polydimethyl Siloxane; SBSE: Stir Bar Sorptive Extraction; HSSE: Headspace Sorptive Extraction; TDU: Thermal Desorption Unit; CIS: Cooled Injector System; MPS: Multi-Purpose Sampler; TIC: Total Ion Chromatogram; DHA: Docosa Hexaenoic Acid; EPA: Eicosa Pentaenoic Acid; DHS: Dynamic Headspace

\section{Introduction}

The majority of customer off-flavor complaints about food and beverage products are probably related to lipid oxidation products (aldehydes, ketones, etc.). Since lipid oxidation problems can cause product recalls and expensive lawsuits, they must be dealt with quickly. This article will discuss examples of lipid oxidation problems and how, in many cases, the lipid oxidation off-flavor contaminants couldn't be readily characterized as originating from lipid oxidation. It will discuss how new technology in sample preparation/extraction coupled with gas chromatographic time-offlight mass spectrometry (GC-TOFMS) instrumentation and peak deconvoluton software can provide insights into the nature of lipid oxidation off-flavors. As the following examples will demonstrate, it is remarkable how many types of foods and beverages are impacted by oxidation, how many unusual ways lipid oxidation contaminants can form, how difficult it is to detect and measure the causative chemicals, and how new analytical techniques are assisting in the elucidation of the oxidation mechanisms involved.

\section{Results and Procedure}

\section{Unprecedented levels of oxidation off-flavor complaints in milk}

Dairy food companies have long been aware of off-flavors caused by butterfat oxidation when milk is exposed to direct sunlight or fluorescent lights in the supermarket dairy case. Companies have gone to great lengths to minimize light exposure from artificial light in supermarkets. A few years ago, the largest domestic fluid milk producer was encountering unprecedented off-flavor complaints in the late winter and early spring. While the problem has been a common industry occurrence, it had been getting worse in recent years. Based on initial sensory testing, the complaints seemed to be related to lipid oxidation even though the company employed packaging to mitigate the problem. Light exposure causes chemical reactions in milk that can modify the proteins and fats to produce many negative flavors, ranging from burnt (burnt feathers or hair) to cardboard or metallic. The resulting 
off-flavors are dependent upon various factors such as exposure time, intensity and wavelength of light exposure, and composition of the milk [1]. Gas Chromatography-mass spectrometry (GC-MS) testing revealed higher levels of hexanal, heptanal and other lipid oxidation aldehydes and ketones had formed in the milk, in addition to elevated levels of dimethyl disulfide, an off-flavor chemical that forms from the degradation of the sulfur-containing amino acid methionine when it is exposed to UV radiation. The aldehydes, ketones and dimethyl disulfide were detected in ppb levels by a technique called headspace solid-phase micro extraction (SPME) GC-MS.

Elevated levels of dimethyl disulfide in all complaints confirmed that light abuse was the cause of the lipid oxidation off-flavors. The critical questions facing dairy technologists: Why were unusually high concentrations of lipid oxidation off-flavors forming in the milk, and how could the problem be eliminated? Chemists used different GC-MS techniques to analyze butterfat profiles. To their surprise, the composition of the butterfat triglycerides was atypical in complaints, containing significantly higher levels of linoleic acid than normal, thus making it more susceptible to lipid oxidation. Further investigation revealed the reason for the change in the fatty acid profile of the milk. During the past few years, dairy farmers were feeding their cows increasing levels of soybeans, a cheap feed source, in their winter rations. This modification in their diet caused modification in the fatty acids profile of milk, increasing levels of linoleic and linolenic acids in the butterfat and elevating levels of malodorous lipid oxidation byproducts. Dairy farmers who supplied milk to the company were told to decrease levels of soybean meal in their winter rations and to add higher levels of vitamin E (antioxidant) supplements to reduce lipid oxidation. Headspace SPME-GC-MS was used to monitor milk from individual dairy farms to ensure compliance.

\section{Vanilla ice cream with "burnt-feathers" off-flavor}

Another interesting problem related to photo degradation of dairy components occurred with vanilla ice cream samples that were stored in a new freezer warehouse facility in Milwaukee, WI. The dairy company began receiving off-flavor complaints (putrid, burnt-feathers malodor) immediately after ice cream samples were shipped to supermarkets from their new freezer warehouse. Food technologists suspected samples were contaminated with a foreign chemical present in the warehouse. However, when they inspected the warehouse, no source could be identified. The complaints continued. GC-MS analysis using a technique called purge-and-trap GC-MS (with Tenax TA as the trapping sorbent followed by GC-MS analysis) revealed the offending chemical was dimethyl disulfide. Protein degradation reactions by microbial enzymes could be a source of the dimethyl disulfide. But high concentrations of hexanal from lipid oxidation reactions in the complaints compared to normal tasting samples from the same lot indicated that the samples may have experienced photo degradation reactions. Other clues supported this theory. For example, the ice cream packaging had a clear plastic lid that allowed light exposure. Not all the samples placed in the warehouse developed the off-flavor.

Food technologists returned to inspect the warehouse a second time - this time looking for potential light sources. The warehouse was equipped with high intensity lights, which were suspected as the cause. It was noted that product stored on the top shelf's in the warehouse were in close proximity to the lights. A new production lot of ice cream was brought into the warehouse. Half of the ice cream on the top shelf was covered with a tarpaulin. Uncovered samples stored on the top shelves developed high levels of dimethyl disulfide (from photo degradation of methionine), while none of the covered samples developed the off-odor. Figure 1 shows chromatograms of a control (normal tasting sample covered by tarpaulin and a complaint uncovered sample with excessive formation of dimethyl disulfide. Technically lipid oxidation wasn't the cause of the off-flavor. However, excessive concentration of hexanal indicated that photo oxidation could be a potential problem because of the degradation of methionine by high intensity lights. This is a good example of how chromatographic testing can be used to supply important indirect information regarding the origins of off-flavor development. In the milk sample discussed in section A, dimethyl disulfide was a confirmatory marker pointing to lipid oxidation products as the cause of off-flavor formation; in this ice cream example, elevation in hexanal levels in complaints was a good marker that the dimethyl disulfide was formed by photo degradation problems with the sulfur containing amino acid.

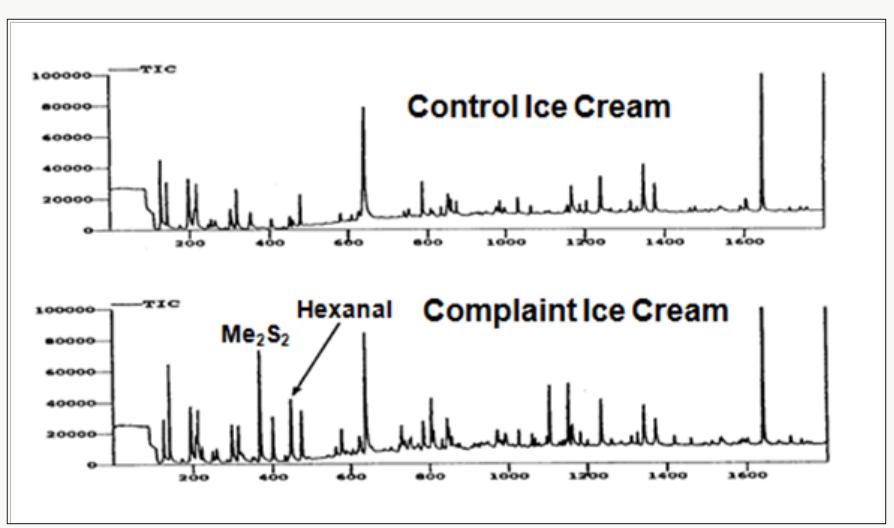

Figure 1: Light-induced putrid off-flavor in vanilla ice cream by purge-and-trap (Tenax TA) GC-MS. 


\section{French onion dip exposed to lights in the supermarket}

Sometimes the problem with resolving lipid oxidation off-flavors is that they're not always easy to identify or are even suspected as a possibility. For example, samples of French onion dip were submitted for analysis because of an unidentified off-flavor. After extensive sensory paneling of ingredients used in the manufacture of the dip, no clear cause was evident. The dip manufacturer considered supermarket lighting could be the source of the offflavor problem but tended to rule it out because the product was sold in light-blocking packaging and the off-flavor wasn't similar to the typical off-flavors that taint oxidized milk. The company resorted to SPME GC-MS testing but couldn't detect any unusual chemical markers typical of lipid oxidation. As a last resort, the food chemists looked to more sensitive GC-MS sample preparation techniques prior to GC-MS. The technique that provided the first clues to the problem was headspace stir bar sorptive extraction (HSSE) GCTOFMS. Figure 2 shows a diagram explaining the experimental steps. To further improve GC-MS analysis, the Leco Pegasus HT time-of-flight mass spectrometer was used to increase sensitivity and provide peak de convolution advantages. A study was set up where normal-tasting control onion dip samples were exposed to UV lights under refrigerated conditions for up to a week of storage. The more sensitive extraction technique detected the tell-tale aldehyde and ketone lipid oxidation products in onion dip samples exposed to UV light for 7 days. HSSE using a PDMS Twister $\rightarrow$ stir bar with 50 times more sorbent phase than a SPME fiber can be significantly more sensitive than SPME, and unlike SPME does not suffer from analyte competition effects with active sorbent sites. As in SPME testing, samples were heated to $50{ }^{\circ} \mathrm{C}$ for an hour during extraction while stirring a mixture of the onion dip and water with a Teflon-coated stir bar. Using a substantially more sensitive extraction technique generated complicated chromatograms with approximately 120 analytes being detected. Overlapping analyte peaks can be a problem. Peak de convolution capabilities become imperative for accurate quantization. The complaint had higher concentrations of all 15 oxidation products compared to control samples. Most significant off-flavor contributing lipid oxidation products were 2-hexenal; heptanal; 2,4-hexadienal; 2-pentyl furan; (E,E)-2,4-decadienal; t-2-nonenal; and nonanal.

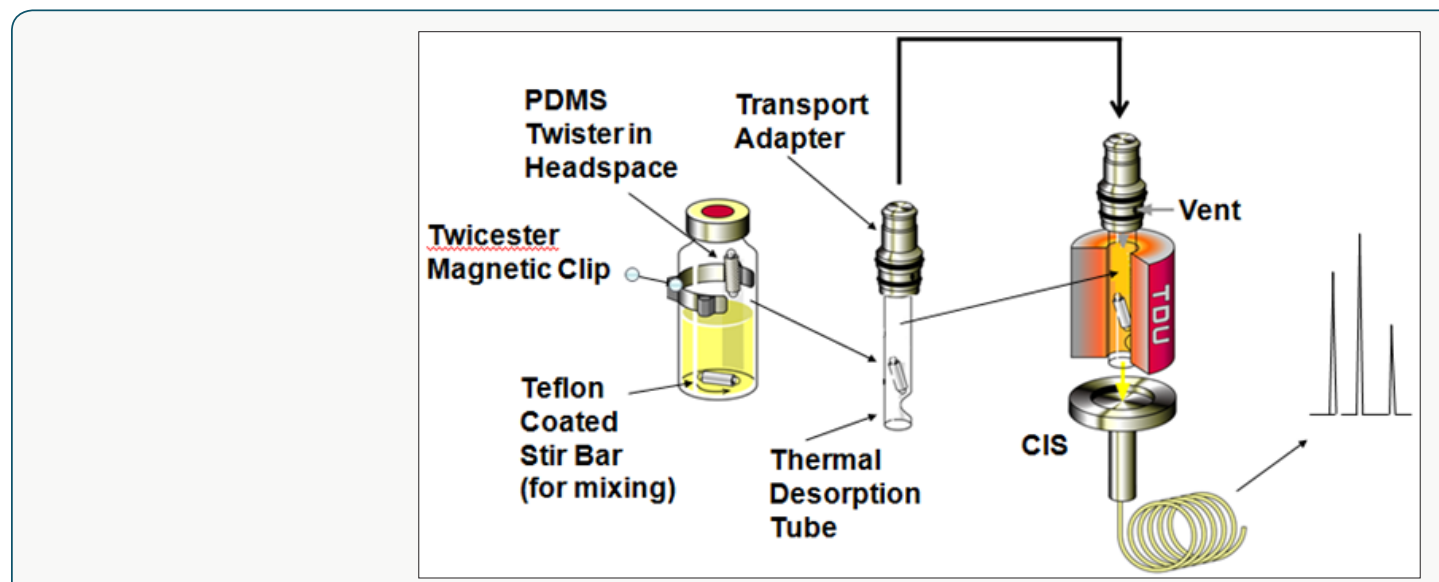

Figure 2: HSSE set up for PDMS Twister extraction using thermal desorption tube, thermal desorption unit (TDU), and cooled injection system (CIS) packed with glass wool.

These chemicals generated the stale taste of the product. Packaging related chemicals detected were styrene (plastic odor) and benzophenone (a photoinitiator used in inks on packaging). However, levels of these two chemicals were too low to be of concern. Approximately 25 different sulfur-containing onion flavor compounds were detected in the samples and were not significantly different in control and complaint sample. Six different lactones were detected in samples with higher levels present in complaint and light-exposed samples vs. controls. These included $\gamma$-caprolactone, $\gamma$-heptalactone, $\delta$-octalactone, $\gamma$-nonalactone, $\delta$-decalactone, and $\delta$-dodecalactone. While low levels of lactones can impart creamy desirable flavors to dairy products, higher levels can impart burnt, fruity, stale, and coconut off-flavors. Lipid oxidation was not initially suspected as the cause of the problems because of the packaging used and because the off-flavor was not recognized as a lipid oxidation off-flavor by sensory panelists
- even though they were trained to identify lipid oxidation offflavors in milk. Background flavor chemicals from the onion flavoring were obscuring the lipid oxidation off-flavors, making them unrecognizable. In addition, a more sensitive extraction GCMS technique had to be used to detect the oxidation malodorants. The chemicals were difficult to extract by other sample preparation techniques because of the high fat content of the product. In addition, the Leco Pegasus GC-TOFMS was required because of its higher sensitivity and peak de convolution capabilities. The fast sampling rates of time-of-flight mass spectrometers generate higher data density than scanning quadruple mass spectrometers, thus providing superior peak de convolution capabilities [2]. Of course, the HSSE GC-TOFMS technique is useful for studying other types of off-flavors in French onion dip and other dairy products. One dip manufacturer was concerned about styrene contamination migrating from packaging and wanted a test that could reliably 
detect less than 5 ppb styrene in the dip. While SPME GC-MS couldn't detect styrene levels at that low of level, HSSE GC-TOFMS was shown to detect as little as $0.1 \mathrm{ppb}$ styrene.

\section{Sour cream oxidation in pilot plant samples}

A food company was experiencing off-flavor problems with a processing line that was used for sour cream production in its pilot plant research facility. It replaced all of the ingredients multiple times and still the problem persisted. Sensory paneling was unable to determine the cause of the off-flavor. Processing temperatures and heat exposure times were carefully monitored. Researchers speculated the problem was related to a microbial culture problem. SPME GC-MS was used to analyze samples. In this case, lipid oxidation aldehydes and ketones were detected but no dimethyl disulfide. While sensory paneling was incapable of recognizing oxidation off-flavors again because of background flavors associated with sour cream, GC-MS analysis clearly showed the off-flavors were caused by lipid oxidation of the butterfat. GC-MS analysis provided even more insight into the cause for the formation of the lipid oxidation byproducts. Photo degradation was not likely the cause since dimethyl disulfide was not detected in the off-flavor samples. Contamination by contact with prooxidant metals was suspected. Inspection of chromatographic peaks identified by mass spectrometry ruled out bad fermentation metabolites. After consultation with the maintenance department that had recently performed "upgrades" to the processing line, food technologists learned that a new copper valve had been installed. After replacing the copper value with a stainless steel valve, the off-flavor problem disappeared. This is a good example of how profiling volatile chemicals with GC-MS can provide useful clues for investigating the source of off-flavor contamination. Using wet chemistry tests like TBARS assay and peroxide value test would not have provided the key clue that dimethyl disulfide was absent and that light oxidation was unlikely.

\section{Detection of oxidation products in nondairy creamer}

A large manufacturer of nondairy creamer (NDC) was experiencing off-flavor complaints with its NDC powder. Sensory analysis pointed to lipid oxidation, a common problem for this product. However, SPME GC-MS could only detect slightly elevated concentrations of hexanal in the complaints compared to the controls but could not detect other unsaturated oxidation chemicals that contribute more potent off-flavors. Again, chemists turned to a different, more sensitive analytical method direct thermal desorption (DTD) using a slitted microvial followed by GC-TOFMS [3]. A slitted microvial with $30 \mathrm{mg}$ of a vegetable oil is shown in Figure 3; for NDC testing, $30 \mathrm{mg}$ of powder was placed in the slitted vial. Compared to the normal non-slitted vial that was typically used, the slitted vial extracted higher levels of volatiles from samples since the carrier gas flow generates more turbulence in the vial to flush out the volatiles analytes resulting in better sensitivity and lower detection limits Figures $4 \& 5$ shows a NDC sample in the slitted vial and in the vial placed in the thermal desorption tube tray of the auto sampler in preparation for thermal desorption in the Gerstel Thermal Desorption Unit (TDU). During the thermal desorption process, volatiles are cryogenically trapped with liquid nitrogen cooling in the Cooled Injector System (CIS). The system is the same as shown in Figure 2, only substituting the thermal desorption tube with a PDMS Twister stir bar with a tube containing the NDC sample in the microvial. The SPME GC-MS test used one gram sample weights of NDC added to 4 milliliters of deionized water and 1 gram of sodium chloride (Figure 6).

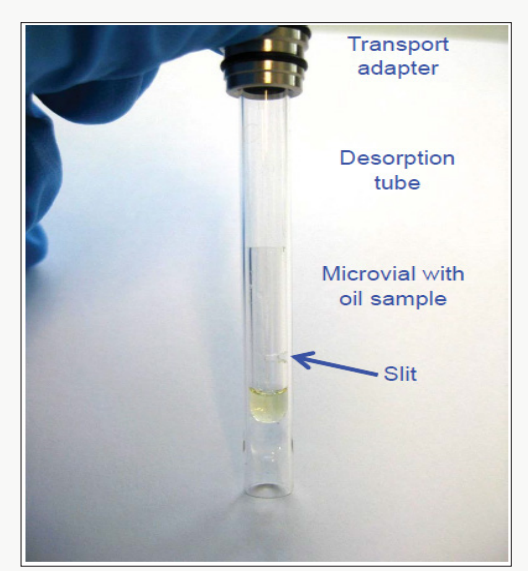

Figure 3: Microvial with slit $1 \mathrm{~cm}$ from bottom of vial with $30 \mathrm{mg}$ edible oil sample for direct thermal desorption (DTD).

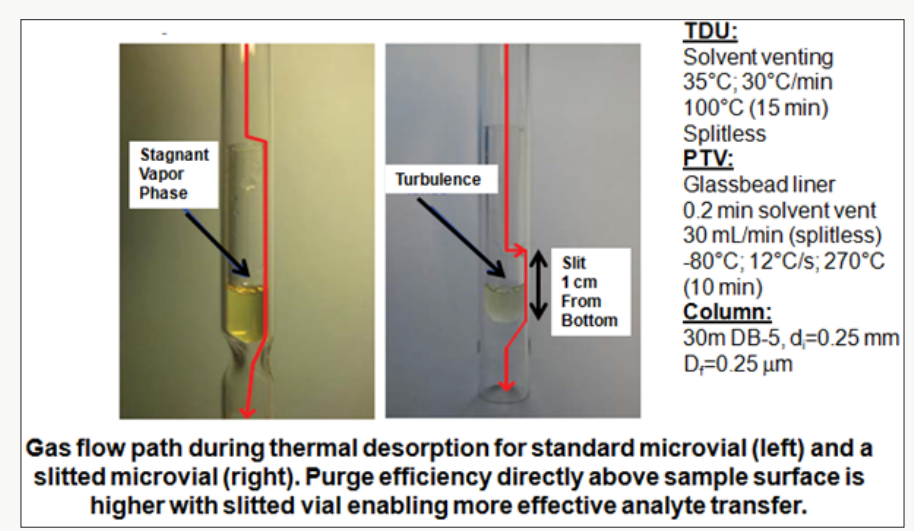

Figure 4: Analysis of edible oil by DTD GC-TOFMS with slitted microvial showing carrier gas flow with increased turbulence. 


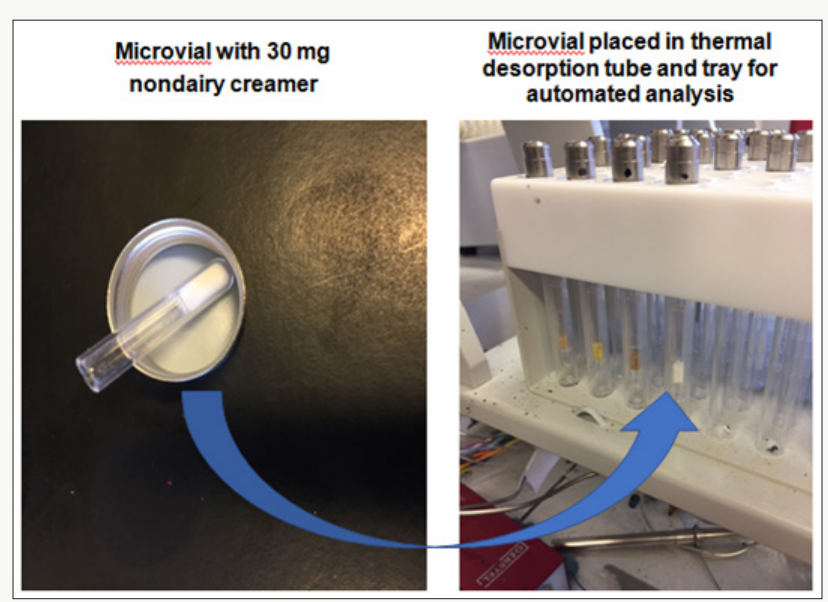

Figure 5: Analysis nondairy creamer by DTD with slitted vial and tube tray holder for automated analysis.

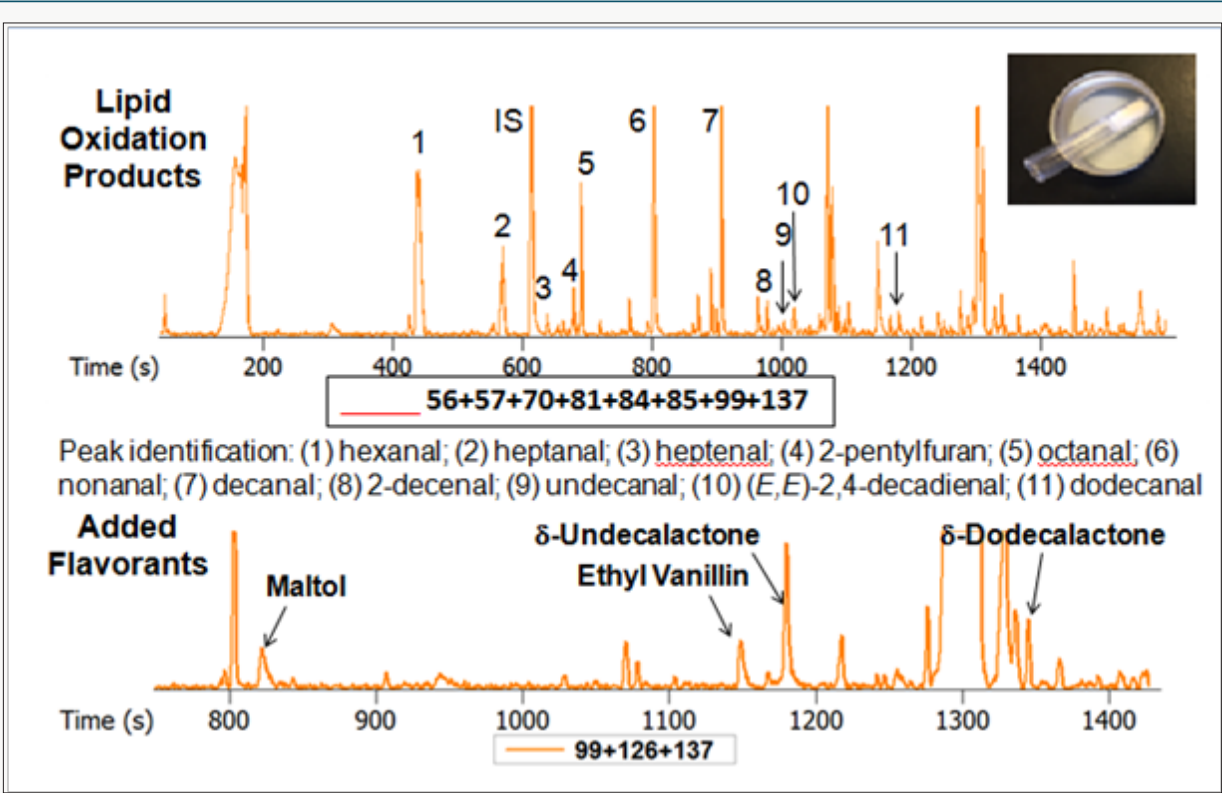

Figure 6: 30 mg nondairy creamer analyzed by DTD GC-TOFMS showing lipid oxidation products and added flavorants.

The sample, water, and salt were added to a $20 \mathrm{~mL} \mathrm{GC} \mathrm{vial,} \mathrm{heated}$ to $50^{\circ} \mathrm{C}$ for $20 \mathrm{~min}$ and extracted with a SPME fiber. Hexanal was the only lipid oxidation product detected. The top trace in Figure 7 shows these results. With the new DTD-GC-TOFMS technique using a slitted microvial and robotic thermal desorption instrumentation from Gerstel Inc., $30 \mathrm{mg}$ of NDC was added to a Gerstel thermal desorption tube. The sample tube was placed in the Gerstel MultiPurpose Sampler (MPS), the auto sampler. The sample was heated to $100^{\circ} \mathrm{C}$ for $15 \mathrm{~min}$, while the eluting volatiles were cold trapped in a PTV injector (Gerstel CIS) cooled to $-80^{\circ} \mathrm{C}$. Once all the volatiles were trapped, the PTV was rapidly heated to $260^{\circ} \mathrm{C}$, releasing the volatiles into the GC capillary column where they could be separated and sent to the TOFMS detector for detection and identification. Even though the DTD sample preparation technique used only $30 \mathrm{mg}$ of sample, it detected eleven different lipid oxidation products that the SPME GC-MS method could not detect. The improved sensitivity of the DTD test vs. SPME is attributed to the fact that DTD testing is an exhaustive volatile extraction technique. SPME is, by nature, an equilibrium extraction technique. Therefore, greater amounts of analytes are extracted by DTD than by SPME. In effect, much higher analyte recoveries are afforded by DTD than by SPME.

The chemists worried that the higher heating temperature used for DTD compared to SPME was generating more lipid oxidation products. They rationalized that increased oxidation was not likely occurring because of the higher heating temperature used in DTD compared to SPME because during thermal desorption of volatiles from the thermal desorption tube; the sample is continually flushed with helium so no air (oxygen) reaches the NDC sample. Additional experimentation, illustrated by the chromatograms in Figure 7 , showed that this, indeed, was the case. A one gram sample of NDC was placed in the $20 \mathrm{~mL} \mathrm{GC}$ vial and heated to $100^{\circ} \mathrm{C}$ for $15 \mathrm{~min}$. The sample was allowed to cool, and the water and salt were added. The sample was then subjected to the normal SPME test. No additional lipid oxidation chemicals were detected in the sample that was 
heated to $100^{\circ} \mathrm{C}$ for $15 \mathrm{~min}$. However, some new chemicals were generated from the heating - furfural and furan methanol from thermal decomposition of sugars in the NDC. These chemicals were not detected in the sample when the DTD method was used, as illustrated in the chromatograms in Figure 6.

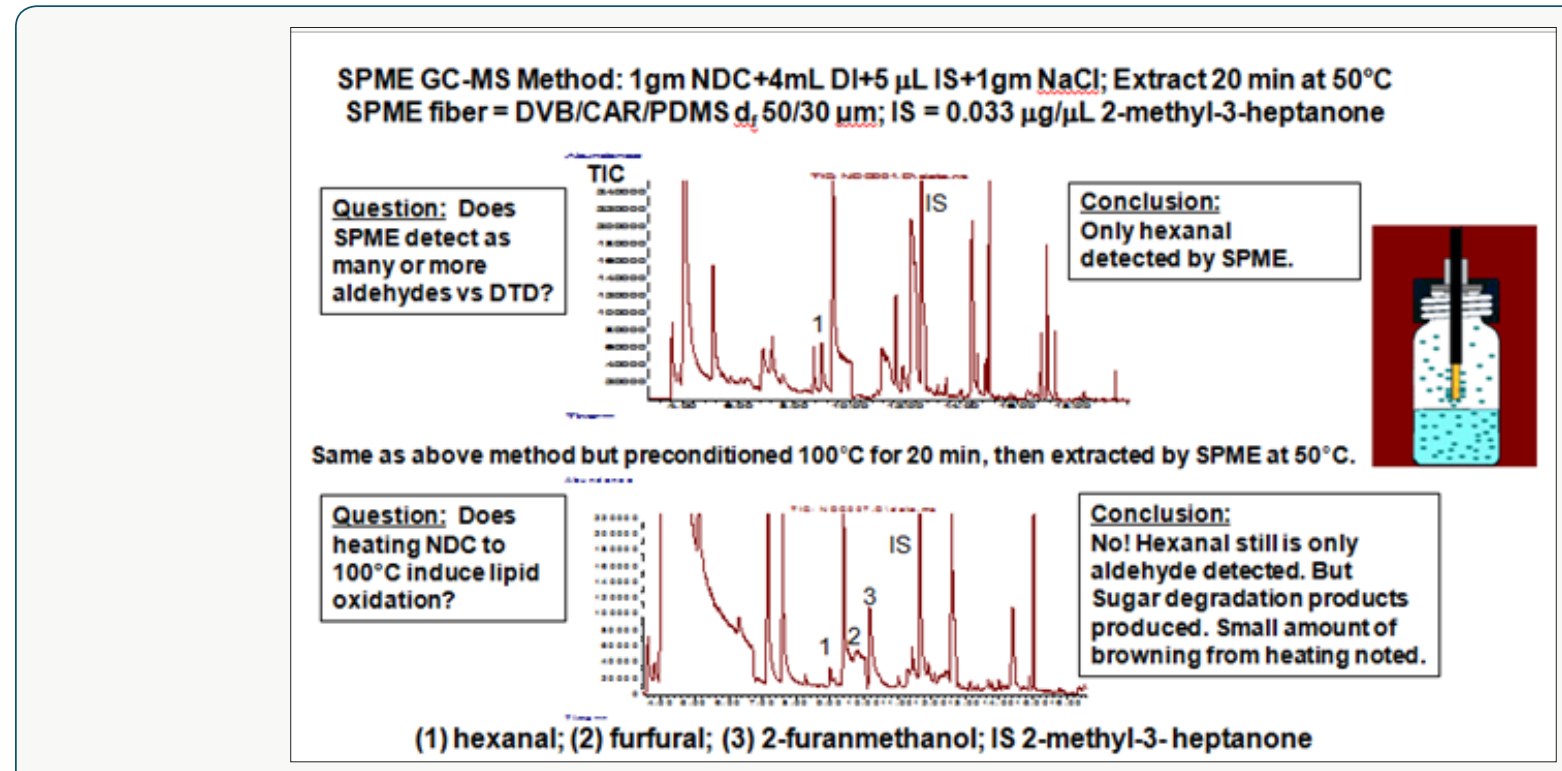

Figure 7: Same nondairy creamer sample as Figure 6 by SPME GC-MS with Agilent 5973 MS.

DTD testing of control and complaint samples with DTD-GCTOFMS (Leco Pegasus HT GC-TOFMS) clearly showed that lipid oxidation products were a source of the off-flavors in the complaint samples. However, it also provided important clues as to the possible origin of these malodorous aldehydes and ketones. In addition to elevated levels of lipid oxidation products, DTD chromatograms revealed elevated levels of free fatty acids and other carboxylic acids (especially hexanoic acid) in complaint samples. These acids likely originated from the casein powder used in the product formulation. Casein powder has been shown to develop a characteristic "wetdog" malodor from microbiological spoilage, generating hexanoic acid and other volatiles acids. Sensory studies with hexanoic acid added to caseinate show positive correlation with a barny/ animal-like sensory attribute [4]. Follow-up studies showed that the lipid oxidation products as well as hexanoic and other acids in the retained caseinate powders were unusually high and were the primary contributors to the malodor of the complaint NDCs. So again, testing with GC-MS was able to not only reveal that lipid oxidation products were contributing off-flavors to the product, but that they were originating from the caseinate ingredient, not the more likely suspect - the vegetable oil used in the product. This is another example of potential of GC-MS techniques to reveal the source of off-flavors in food products.

\section{Complaint with bottled water samples}

Complaint and control bottled drinking water samples were submitted for analysis. Customers complained the water samples had a malodor reminiscent of motor oil. SPME-GC-MS, a common technique now employed in food industry laboratories for flavor studies, was used for testing volatiles. In this case, an olfactometry detector was added to the analytical instrumentation. With this accessory, the chemist injects a sample extract into the GC injector. The mixture of volatiles passes through the column, separate into component peaks, and exit the column. Half of the effluent is directed to the MS detector for peak identification and peak intensity measurement, while the other half goes to the olfactometry detector. Chemists or sensory scientists can then sniff each peak as it elutes from the column and determine the odor characteristics of each eluting peak. SPME GC-MS analysis of control and complaint samples did not reveal any significant chemical differences in the samples or any significant odors by olfactometry. The samples were reanalyzed using stir bar sorptive extraction (SBSE) GC-MS, a technique employing polydimethyl siloxane (PDMS) sorbent as an extract ant that can be 1000 times more sensitive than SPME-GCMS. It is further explained in Figure 8. SBSE is based on the Gerstel Twister $\rightarrow$ (Baltimore, MD). For these samples, the $2 \mathrm{~cm}$ PDMS Twister with $0.5 \mathrm{~mm}$ film thickness ( $63 \mu \mathrm{L}$ volume) was used to increase analyte sensitivity. Thirty millilters of sample was stirred for $2 \mathrm{hr}$. As shown in Figure 8, hexanal, heptanal, octanal, nonanal and decanal-all potential lipid oxidation products-were detected in all complaint samples but not in any of the control samples (or were at least at levels 10 higher in complaints vs. controls). Only hexanal was detected by SPME GC-MS even though the same type of GC column and chromatographic conditions were used. Flavor chemists know that this group of aliphatic aldehydes are potential lipid oxidation products but certainly don't smell like motor oil. Furthermore, when the samples were injected into the GC-MS using SBSE-GC-MS, no odors were detected, except for octanal which had a slightly sweet/green odor. No other chemicals clues were present that could explain the cause of the malodor in the complaints. More testing that was conducted. 


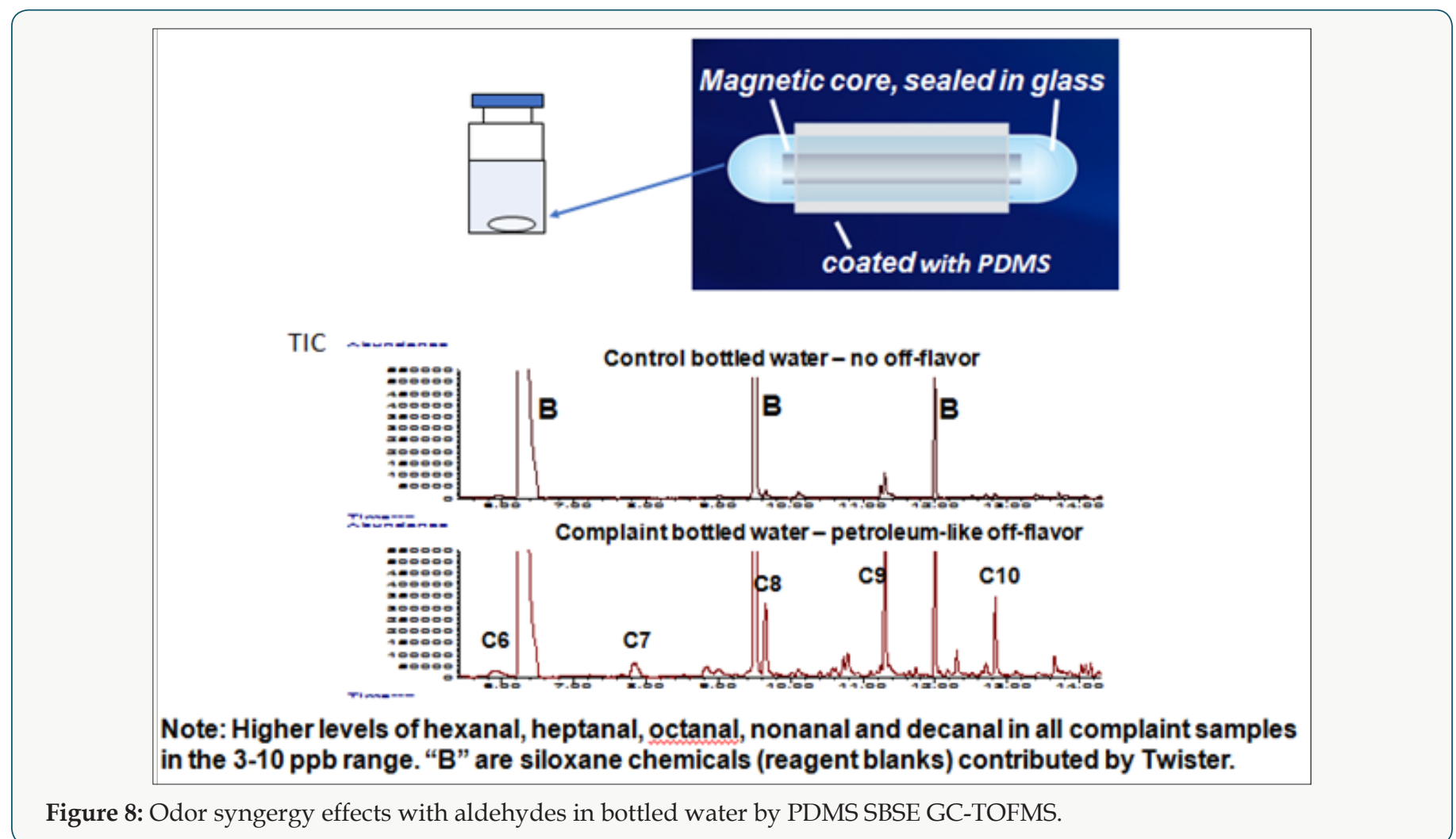

In every case, complaint waters had significantly higher levels of aliphatic aldehydes than controls. Aldehydes were the only significant peaks detected. On a hunch, the chemists considered possible odor synergy effects. Combinations of single chemicals can produce enhancing or masking effects odor sensory effects. In addition, cases of odor synergy effects have been known to create odors unrelated to the individual contributing chemicals, as explained in the next example for fishy malodors. Researchers conducted experiments to determine if various combinations of aldehydes smell like motor oil even though the individual aldehyde components did not [5]. When odor synergy effects are observed, combinations of two or more chemicals can generate odors that are total different and unrelated to the individual compounds when they are smelled individuals. To test if this was the case, chemists conducted a model systems study-subjecting a series of standards containing various combinations and concentrations of the aldehydes in DI water to sensory analysis. They were able to determine that a mixture of octanal and nonanal $(>4.0 \mathrm{ppb}$ and $>6.0$ ppb, respectively) generated the identical motor oil malodor. This was astonishing to the chemists and sensory scientists involved in the study since these well known chemicals had never been associated with a motor-oil type malodor. Figure 8 shows SBSE GC-TOFMS total ion chromatogram (TIC) results for the sensitive SBSE technique. One baffling question: What was the source of the lipid oxidation products present in bottled water-a product with no added unsaturated fat? Further investigation revealed the source of the aldehydes was photo degradation of erucamide, the amide of C22 mono-unsaturated erucic acid, the slip agent used in closures of the bottled water samples.

\section{Omega-3 fortification causing fishy malodors}

While oxidation reactions of docosahexaenoic acid (DHA) and eicosapentaenoic acid (EPA) are responsible for fishy off-flavors in marine oils, gas chromatography-olfactometry (GC-O) and other types of analytical studies have failed to reveal which specific oxidation products are involved - until recently. GC-MS-O studies of marine oils with fishy malodors revealed approximately 32 lipid oxidation products irrespective of the analytical extraction technique used including SPME, dynamic headspace (DHS), or HSSE, but none were characterized as fishy. However, when all sample volatiles were captured together and then desorbed simultaneously in GC-O experiments, the fishy malodor was evident, indicating odorant synergy effects were responsible. By trapping various combinations of lipid oxidation products and then eluting them in GC-O experiments researchers were able to identify that the combination of two lipid oxidation products - heptanal and (E,Z)-3,5-octadien-2-one were responsible for the fishy malodor that develops with omega-3 products. Excluding either heptanal or (E,Z)-3,5-octadien-2-one eliminated the fishy malodor from omega-3 products [6]. To prove that heptanal and (E,Z)-3,5octadien-2-one were synergistically involved with creating fishy malodor, researchers just trapped these two chemicals from a sample and released them simultaneously to the olfactometry port. A strong fishy malodor was observed. Next, they tried omission experiments. They injected a sample with fishy malodor and trapped all chemicals accept heptanal. When all chemicals minus the heptanal were released to the olfactometry detector, no fishy odor was detected. They repeated the experiment, but this time 
eliminating only the (E,Z)-3,5-octadien-2-one. Again no fishy malodor was detected.

\section{Conclusion}

The examples discussed in this report, show that detection of lipid oxidation products in complex samples and determining their mechanism of formation can be challenging. However, with the aid of modern innovative extraction techniques like SBSE, HSSE and DTD in combination with highly sensitive TOFMS detectors (e.g., Leco Pegasus HT TOFMS or the Leco BT TOFMS), it is possible to decipher critical causes of lipid oxidation off-flavors in foods and beverages so adjustments in ingredients, processing conditions or sample storage, can be made quickly to avoid product recalls and damage to a product's reputation.

\section{References}

1. Marsili RT (2011) Flavors and Off-Flavors in Milk and Dairy Products. In Fuquay JW, Fox PE, McSweeny PHL (Eds.), Encyclopedia of Dairy Chemistry $2^{\text {nd }}$ (edn.) Academic Press, London, pp. 533-551.
2. Holland JH, Gardner BD (2001) The Advantages of GC-TOFMS for Flavor and Fragrance Analysis. In Marsili RT (Eds.), Flavor, Fragrance and Odor Analysis. Marcel Dekker, Inc, New York, USA, pp. 107-132.

3. Gerstel, Baltimore, Appnote (2014) Analysis of Aroma Compounds in Edible Oils by Direct Thermal Desorption GC/MS Using Slitted Microvials.

4. Karagul Yuceer Y, Vlaovich K, Drake M, Cadwallader K (2003) Characteristic Aroma Components of Rennet Casein. J Agric Food Chem 51(23): 6797-6801.

5. Marsili RT (2014) The importance of Odourant Synergy Effects in Understanding Malodor Problems in DHA and EPA Products. Lipid Technology 26(2): 31-34.

6. Marsili RT, Laskonis CR (2014) Odorant Synergy Effects as the Cause of Fishy Malodors in Algal Marine Oils. J Agric Food Chem 62(40): 96769682.

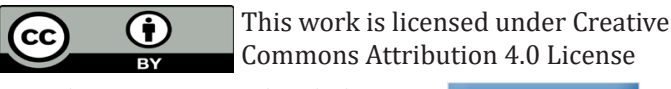

To Submit Your Article Click Here: Submit Article

DOI: 10.32474/CIACR.2018.02.000131

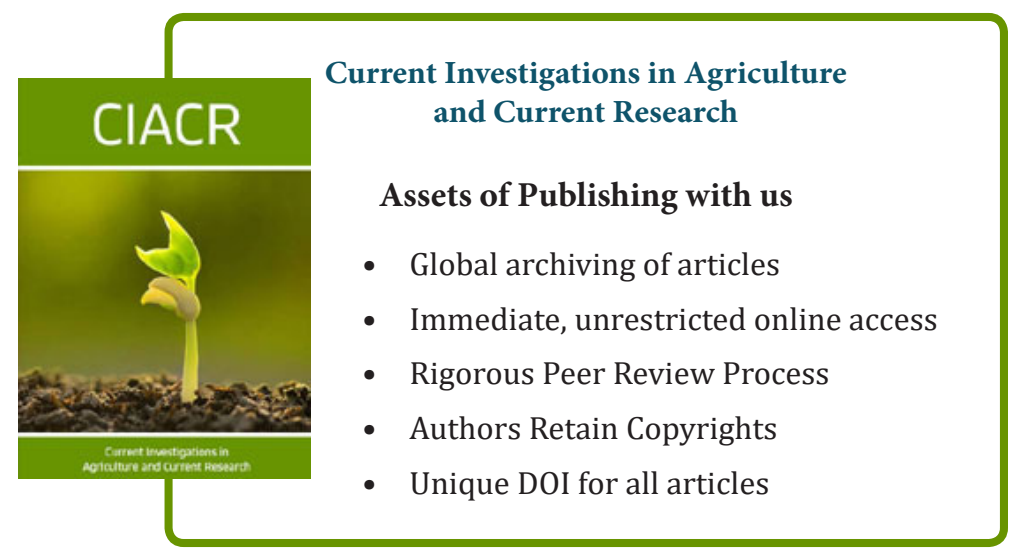

